\title{
CATALOGUE OF ZERYNTHIINAE AND ALLIED GENERA IN THE TRING MUSEUM, WITH CRITICAL NOTES.
}

\author{
By LORD ROTHSCHILD, F.R.S., Pн.D.
}

SINCE I compiled the catalogue of the genus Parnassius in the Tring Museum

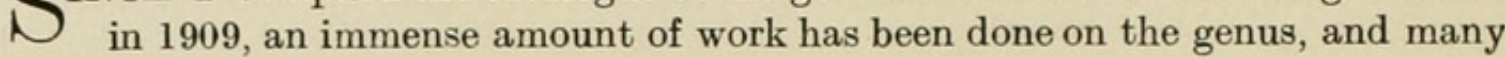
articles have appeared, among the principal authors being Herr Fruhstorfer, Dr. Verity, Mr. Adolph Bryk, and the late Dr. Pagenstecher. Monsieur Avinoff, also, has made great collecting trips in many parts of Central Asia and has made numerous fine discoveries. Though very few new species have been described, a host of new local races have been discovered; and not a few, which were previously known, have, owing to more careful investigations, proved to be distinct from the races they had previously been identified with. The number of specimens of Parnassius at Tring has increased very largely in the last nine years, but not in all the forms. I have lately rearranged the genus and co-ordinated it with the allied genera, and I think, owing to the great interest taken in these fine insects, a new and revised catalogue is not superfluous. I am including all the genera allied to Parnassius and Papilio as well as Parnassius itself in the two articles I am publishing in this volume. I gave a list of Parnassius only in Novitates in 1909. Except in very few instances I am not enumerating the individual specimens of aberrations, only stating that among the enumerated series are individuals of named aberrations.

The order of the subspecies, and sometimes even of the species, is not always that followed by Herr Stichel or by Dr. Verity, and must not be taken as meaning that $I$ assert that their classifications are wrong; but merely as being one I found easier to follow in arranging my series.

\section{IUEHDORFIA.}

Luehdorfia puziloi (Ersch.).

Luehdorfia puziloi puziloi (Ersch.).

Thais puziloi Erschoff, Horae Soc. Entom. Ross. vol. viii. p. 315. No. 1 (1871) (Ussuri River).

8 ธิธิ, 6 우. No definite locality; 2 우 Tjutzu-ho, 400 kilometres N. of Wladiwostock, June 1909 (W. Mau).

Luehdorfia puziloi yessoensis subsp. nov.

This form has always been placed as identical with $p$. puziloi, but it is quite distinct.

๙. Differs mainly in the much heavier and wider dark border to the hindwings and the black bands of the forewings are heavier. of shows the same differences from $p$. puzilo $i$ as the $\delta$ does, but in addition the median vertical lappet of the horny pouch is much larger and longer.

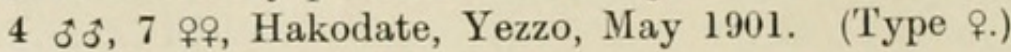


Inehdorfia japonica Pryer.

I cannot agree with Staudinger and Stichel that $L$. japonica is only a subspecies of $L$. puziloi, for the horny pouch of the $q$ is totally different. In puziloi this pouch is winged and in shape somewhat like a butterfly, and along the centre runs an upright flange or ridge developing behind into a free lappet and coloured rich chestnut-brown. In japonica on the other hand the pouch is almost circular, sharply truncated in front, having in the centre a broad raised roof-like structure resembling a tent or penthouse, the whole coloured deep black or deep black-brown. I therefore conclude that L. japonica is a good distinct species. It has three races and occurs in China, Japan and Formosa.

\section{Luehdorfia japonica japonica Pryer.}

Luehdorfia japonica Leech, Entomologist, vol. xxii. p. 25. pl. I. f. 1 (1889) (Gifu).

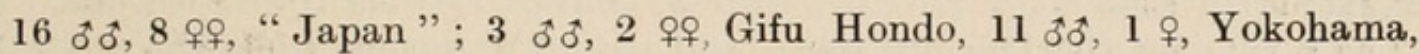
Japan. 2 larvae and 1 pupa, Gifu.

Luehdorfia japonica formosana subsp. nov.

Di ers from $j$. japonica in having the black markings on the forewings more extended and the yellow bands narrower.

2 đิ ${ }^{*}$, Formosa (bought of Ernest Swinhoe, 1905).

Luehdorfia japonica chinensis Leech.

Luehdorfia japonica var. chinensis Leech, Butt. Chin. Jap. and Cor. vol. ii. p. 491. t. 33. f. 1. ㅇ. (1893) (Central China).

Dr. Staudinger calmly united $J$. j. chinensis with $L . j$. japonica as a synonym while not even quoting China as a habitat of japonica. The true facts of the case are that $L . j$. chinersis is an abundantly distinct subspecies. Differs from $L . j$. japonica in its deep yellow ground-colour, very narrow black markings, and especially in the red submarginal band of the underside of the hindwings being present on the upper side; whereas in all other Luehdorfias there is on the upper side only a subanal red patch above the tornus.

The horny pouch is similar to that of $j$. japonica but smaller, and the tentlike erection is much less developed.

1 ơ, Lu-Shan Mountains, Kiukiang ; 1 \%, Chang Yang, 6,000 ft. 1889 (Nat. Coll.), ex coll. Leech, ex coll. British Museum in exchange.

\section{EURYADES.}

\section{Euryades duponcheli (Lucas).}

Papilio duponcheli Lucas, Ann. Soc. Entom. France, vol. viii. p. 93. pl. 8. f. 1. (1839) (Entre Rios).

4 ôे, Estancia Cooper, Alto, Paraguay (Th. Inslay); 1 ô, 1 † Sapucay, Paraguay, May 1902-November 1903 (W. Foster); 1 ㅇ Rio Burmejo-Rio

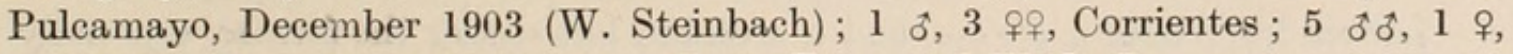
Tucuman, January to April, 1901 (G. A. Baer and W. L. Reeve) ; 3 ô ô, 3 우, Paraná, January 1897 (Ruscheweyh) ; 15 ô ô, 5 우, Suncha Corral, Santiago del Estero, Argentina (J. Steinbach) ; 1 ô, 1 f, La Rioja, Argentina (E. Giacomelli) ; Belgnano, Buenos Aires, March 1897 (Ruscheweyh); 1 ô, 1 우, Entre Rios (Burmeister), ex Felder coll. 


\section{Euryades corethrus (Boisd.).}

Papilio corethrus Boisduval, Hist. Nat. Ins. Spec. Gén. Lépid. vol. i. p. 314. No. 152. pl. 17. 1. C. (1836) (Patr. Tgn.).

4 ô ${ }^{*}, 3$ 우, Sapucay, Paraguay, January 1899, August 1093, November

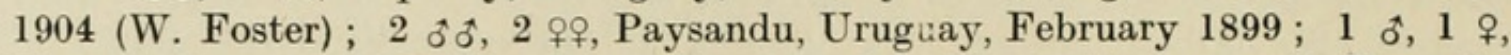
Paraná, January 1895 (Ruscheweyh) ; 1 ô, 1 \&, Montevideo, ex coll. Burmeister, ex coll. Felder.

\section{EURYCUS.}

\section{Eurycus cressida (Fabr.).}

I cannot agree with Dr. Jordan that the Eurycus from the S. West and neighbouring islands are identical with $E$. cressida cressida from Australia. The 우 as a rule are considerably larger and more diaphanous while the outer $\frac{1}{3}$ of hindwings is paler and less scaled.

\section{Eurycus cressida cressida (Fabr.).}

Papilio cressida Fabricius, Syst. Entom. p. 448. No. 24 (1775) (New Holland, Mus. Banks).

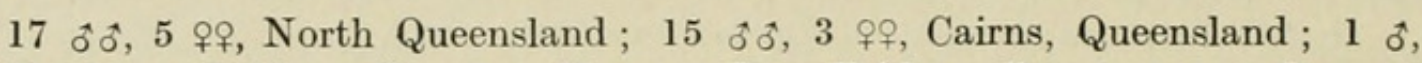
1 o, Brisbane, Felder coll. ; 1 ô, Moreton Bay, Felder coll. ; 1 ô, 1 , Queensland, Barnard coll. 1 pupa, North Queensland, 2 pupae and 1 pupa shell, Townsville, Queensland (Dodd).

\section{Etrycus cressida kühni subsp. nov.}

Differs from $c$. cressida chiefly in the 우. ㅇ more diaphanous, generally larger, the scaled portions paler and yellower and less extended on hindwing.

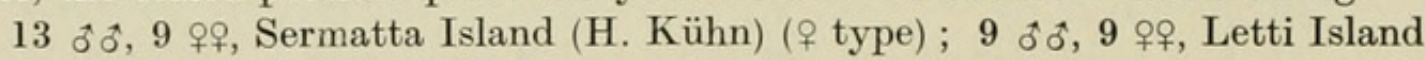
(H. Kühn) and (W. Doherty, July 1892); 17 ơ ô, 6 우, Moa Island, December 1902 (H. Kühn) ; 6 oิ ô, 7 우, Kisser Island (H. Kühn) ; 2 oิ ô, Babber Island (H. Kühn).

\section{Eurycus cressida troilus Butl.}

Eurycus troilus Butler, Ann. Mag. Nat. Hist. (4) xviii. p. 247. No. 27 (1876) (Port Moresby).

$1 \hat{o}, 1$, Redscar Bay, British New Guinea, 1894 (Lix) ; 1 $\star$, Mailu, British $\mathrm{N} \in \mathrm{w}$ Guinea, July 1895 (Anthony) ; 4 우, Port Moresby (Rattle) ; 1 ๙ Lower Aroa River, British New Guinea, November 1904-March 1905 (A. S. Meek).

\section{Eurycus cressida intermedius subsp. nov.}

Differs from both $c$. troilus and c. cressida, but nearer the former, especially in the q. of almost identical with $c$. cressida. ㅇ. Above similar to c. troilus o, but the discal white patches on the hindwing larger; below has the white marginal spots of $c$. cressida.

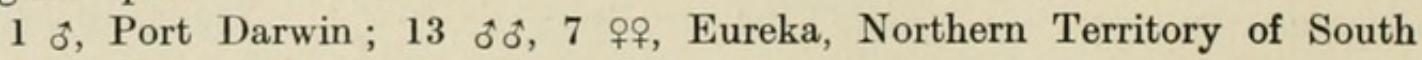
Australia, January 1903 (Tunney). 
BARONIA.

Baronia brevicornis Salv.

Baronia brevicornis Salvin, Trans. Entom. Soc. London, 1893, p. 331 (Chilpancingo, W. Mexico).

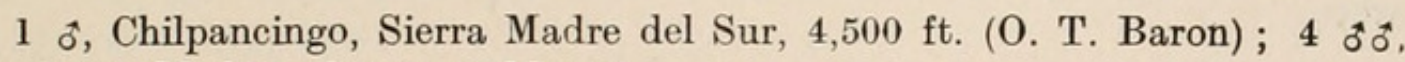
3 오, Sierra Madras, Guerrero, Mexico.

\section{IEPTOCIRCUS.}

\section{Ieptocircus curius (Fabr.).}

There are 3 subspecies all very closely allied.

Leptocircus curius curius (Fabr.).

Papilio curius Fabricius, Mant. Insect. vol. ii. p. 9. No. 71 (1787) (Siam, ex Mus. Banks).

2 우, Silhet (1 ex coll. Feld.); 1 ơ, Khasia Hills, Assam; 1 ㅇ, Assam, coil. Felder; 1 , Katha, Burma ; 1 ô, Burmah; 1 \&, Siamese Frontier of

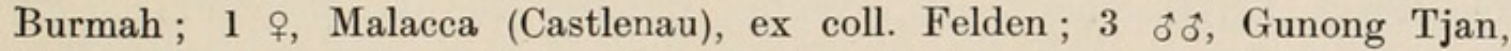
Malay Peninsula; 1 ô, 2 우, Mount Jahan, Malay Peninsula (Waterstradt) ; 1 ; Namktan-Bhamo, Northern Shan States; 1 ; Sumatra; 1 ;, Upper Palembang District (Völcker); 1 ô, Padang Sidemi, W. Sumatra (Erichson) ; 1 ơ, 1 †, N.E. Sumatra, December 1892 (Dr. Martin); 3 ô ô, Selesseh, N.E. Sumatra, June 1893 (Dr. Martin); 1 ô, 5 우, Battak Mountains, N.E. Sumatra, June 1893-May 1894 (Dr. Martin); 3 oิ oิ, 2 우, Kina Balu (1 ô, A. Everett), 2 ơ ô, 2 우우, December 1898-February 1899 (Waterstradt); 1 우, Lawas, N. Borneo (A. Everett); 1 ô, 1 ㅇ, Mount Marapok, Dent Province,

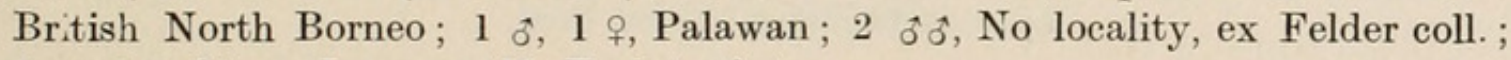
1 o $\$$, 1 o , Siam, January (H. Fruhstorfer).

\section{Leptocircus curius walkeri Moore.}

Leptocircus walkeri Moore, Lépid. Ind. vol. v. p. 137 (1901-1903) (Hong Kong).

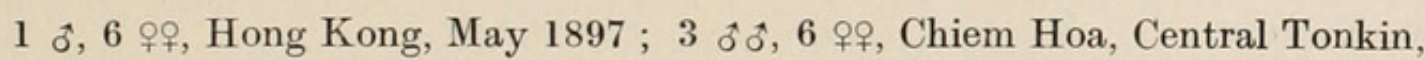
August-September (H. Fruhstorfer) ; 1 o, 14 \&ᄋ, Youboi, Hainan, June 1904 ; 5 우, Mangrin, Hainan, June 1904 ; 1 oै, 3 우, Henron, Hainan, June 1904; 1 。, Hainan, 1904-1905.

Leptocircus curius libelluloides Fruhst.

Leptocirus curius libelluloides Fruhstorfer, Berl. Entom. Zeitschr. vol. xliii. p. 179 (1899) (Nias).

3 ơ ô, 4 우오, Nias.

\section{Leptocircus meges (Zink.).}

Of this species 5 subspecies can be distinguished.

\section{Leptocircus meges meges (Zink.).}

Papilio meges Zinken, Nova Acta Acad. Nat. Cur. vol. xv. p. 161. pl. 15. f. 8 (1831).

8 ôे, 1 \&, Battak Mountains, N.E. Sumatra, June 1893-November 1894 (Dr. Martin) ; 1 ơ, 1 \&, Selesseh, N.E. Sumatra, November 1893-March 1894 (Dr. Martin) ; 4 와, Setinjak, W. Sumatra, February-May 1898 (Erichson); 
1 ô, Gayos Mountains, N.E. Sumatra, June 1903 (Dr. Martin) ; 3 ㅇ, Mount Mulu, 1,000-4,000 ft., N. Borneo, August-December 1894 (Charles Hose);

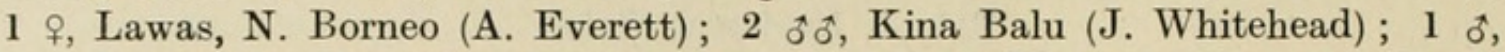
1 \&, Kina Balu, December 1898-February 1899 (J. Waterstradt) ; 1 ơ, South Borneo.

\section{Leptocircus meges virescens Butl.}

Leptocircus virescens Butler, Cat. Diurn. Lepid. descr. Fabr. p. 259 (1870) (Java and Moulmein).

5 ơ ơ, 5 우우, Chien Hoa, Central Tonkin, August-September (H. Fruhstorfer) ; 1 ; , Tonkin, June-July (H. Fruhstorfer); 3 Burma (Fea) ; 1 ;, Mepli Valley, December 1893 (Colonel Bingham); 1 ; , East Pegu, 500-2,000 ft., March-April, 1890 (W. Doherty); 1 ô, 1 ㅇ, Dawnatt Range, September 1891 (Colonel Bingham ); 1 o, Van Bu, Upper Tonkin ; 2 우, Shan States ; 1 ㅇ, Moulmein, September 1892 (Colonel Bingham); 1 đo, Pukha, Siamese Frontier, S. Shan States, 2,000 ft., February 1895; 1 ㅇ, Haundraw, June 1895 (Colonel Bingham) ; 1 o, Mount Tahan, Malay Peninsula (J. Waterstradt) ; 1 ô, NamHou (Black River), Siam-Tonkin Frontier, October 1905 (W. Micholitz); 1 ;, Xom-gom, S. Annam, February (H. Fruhstorfer) ; 1 ô, 2 우, Malay Peninsula ; 2 우, Malacca (Castlenau), ex coll. Felder ; 3 ธิ ô, 3 우, Youboi, Hainan ; 2 우, No locality, ex Felder coll.

\section{Leptocircus meges niasicus Jord.}

Leptocircus meyes niasicus Jordan, Seitz Grossschm. Erde, vol. ix. p. 108 (1909) (Nias).

1 ค, Nias Island.

\section{Leptocircus meges decius Feld.}

Leptocircus decius Felder, Wien. Entom. Monatsb. vol. vi. p. 284. No. 37 (1862) (Luzon).

1 oै, 1 ㅇ? ?; 4 우, Palawan ; 1 ô, Balabac ; 1 ㅇ, Luzon (Lorquin) (type), ex.

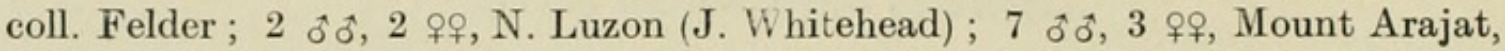
Luzon, August 1903 (Browne); 6 우, Mindoro, November-December 1894 (A. Everett) ; 1 \%, Mindoro, bought from Staudinger.

\section{Leptocircus meges ennius Feld.}

Leptocircus ennius Felder, Reise der Novara, Lepid. vol. i. p. 2. tab. 21. b. (1865) (Menado).

1 ․, Menado, 1 ㅇ, Celebes (Wallace), 1 ㅇ, no special label, 1 우, Macassar, April 1862 (type), ex coll. Felder; 1 o, 1 \&, Dongala, S. of Palos Bay, Celebes, August September 1896 (W. Doherty) ; 1 \%, Tavoya, N. of Palos Bay, Celebes, August 1896 (W. Doherty); 1 ô, North Celebes.

\section{TEINOPALPUS.}

Teinopalpus imperialis Hope.

In 1908 (Novitates Zoologicae, p. 602) I described the Bhutan and Sikkim individuals of this species as a distinct race and named it T. imp. himalaicus. In 1909 Dr. K. Jordan, in Seitz Grossschm. Erde, vol. ix. p. 108, sinks this as a synonym of $T$. imperialis imperialis Hope, saying that it was true that Bhutan and Sikkim 우 had the underside of the hindwings paler than Assam specimens, but that equally pale ones occur occasionally among Assamese 우. In view 
of this I should have agreed with him and included these Bhutan-Sikkim specimens under imperialis; but there is one point of difference that seems constant, namely, the supra-tornal yellow patch, both above and below, is larger and extends further into the wing than in any Assam 우 $\mathrm{I}$ have seen, many amongst which have this patch much reduced and almost absent. I thus recognize 3 subspecies.

Teinopalpus imperialis imperialis Hope.

Teinopalp's imperialis Hope, Trans. Linn. Soc. vol. xix. p. 131. pl. 11. f. 1, 2 (1843) (Silhet).

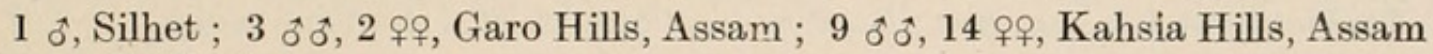
(1 o ex coll. Felder).

Teinopalpus imperialis himalaicus Rothsch.

Teinopalpus imperialis himalaicus Rothschild, Novit. Zool. vol. v. p. 602. No. 1. (1898) (Bhutan).

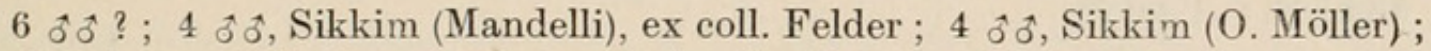
1 ô, Ari-Gnaton Sikkim, 7,000-12,500 ft., April 1892 (Colonel Bingham) ; 1 \&, Bhutan, May 10th, 1889 (J. G. Pilcher) ; 1 \%, Darjeeling, May 1889 (J G. Pilcher ?).

Teinopalpus imperialis imperatrix Nicév.

Teinopalpus imperatrix De Nicéville, Journ. Bombay Nat. Hist. Soc, vol. xii. p. 335. No. 47. pl. BB. ơ우 (1899) (Tenzsserim).

2 ô ô, 1 ㅇ, Taungou Hills, 4,000 ft., Upper Tenasserim, ô January, ㅇ March (T. A. Hauxwell) (types).

\section{ARIIANDIA.}

Armandia thaidina Blanch.

Armandia thaidina Blanchard, Compt. Rend. vol. Ixxii. p. 809. Note 3 (1871) (Mou-pin).

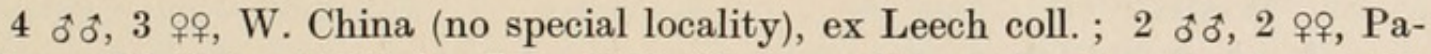
tsu-fong, W. China, 9,820 ft., June 1890 (Native coll.), ex Leech coll. ; 1 ơ, 4 우, Wa-ssu-kou, 5,000 ft., June 1890 (Native coll.), ex Leech coll. ; 1 đ, 1 우, Washan, W. China, 6,000 ft., June 1889 (A. E. Pratt), ex Leech coll. ; 1 , W. China (no precise locality), ex Leech coll., ex Felder coll.

\section{Armandia lidderdalii (Atk.).}

Atkinson in describing this very remarkable insect was unaware of the previous description of thaidina by Blanchard, and founded on it the genus Bhutanitis. Those genus splitters who go entirely by shape and outline maintain the two genera, but structurally Dr. Jordan could find nothing to warrant the separation. There are two subspecies, one only being in t.e Tring Museum.

\section{Armandia lidderdalii lidderdalii (Atk.)}

Bhutanitis lidderdalii Atkinson, P.Z.S. 1873. p. 571. pl. 50 (Bhutan).

4 ธิธิ, 1 \&, "Bhutan" (no locality on labels); 4 oิ Bhutan ; 3 ơ ô, 1 ㅇ, Bhutan ; 1 ơ 4 q̊, Bhutan, September 1888 (J. G. Pilcher) ; 2 ỡ , S. Chin Hills, Burma, 6,000-7,000 ft., August $1895 ; 3$ ô , Sikkim. 


\section{SERICINUS.}

There is only one species of this genus but a number of subspecies; each p.obably appearing in two seasonal forms, of which the spring generation of 3 only have been described.

\section{Sericinus telamon (Don.).}

There are six subspecies of this insect.

Sericinus telamon telamon (Don.).

Papilio telamon Donovan, Epit. Nat. Hist. Ins. China, pl. 27. f. 1 (1798) (Pekin).

Gener. Vern. telmona Gray.

Sericinus telmona Gray, P.Z.S. 1852, p. 72 (N. China).

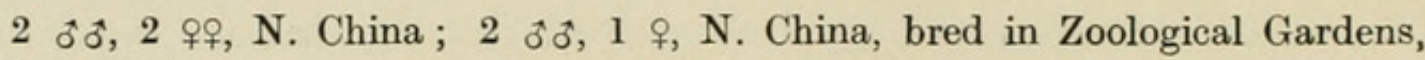
London; 1 larva.

Gener. Aestiv. telamon Donovan.

6 ô ô, 4 우, N. China ; 1 ô, 1 ㅇ, N. China, ex Felder coll. ; 1 ô, Pekin, Tatarinoff, 1 ô, Ningpo (Muirhead), ex Felder coll.; 1 o, 1 \%, Tsingtau, Shantung (L. Klapheck) ; 1 ô, 1 ㅇ, Pekin, September-November, 1902 (Colonel Browne); 6 ธิ oิ, 2 우, Chifu, Shantung, N. China ; 1 ô, Wei-hai-Wei, September 1898 (Dr. Lambert); 1 \&, Talhienteze, N. China.

\section{Sericinus telamon amurensis Stdgr.}

Sericinus telamon var. amurensis Staudinger in Romanoff, Mém. Lépid. vol. vi. p. 130 (1892) (Sutschan District).

Gener. Vern. telemachus Staudinger.

Sericinus telamon var. amurensis gen. verı. telemachus Staudinger in Romanoff, Mém. Lépid. vol. vi. p. 133 (1892).

4 ธิธี, 6 우, " Amur."

Gener. Aestiv. amurensis Staudinger.

3 ธิธิ, 2 우우, "Amur."

\section{Sericinus telamon koreana Fixs.}

Serecinus telamon var. koreana Fixsen in Romanoff, Mém. Lépid. vol. iii. p. 257 (1887) (Korea).

\section{Gener. Aestiv? koreana Fixs.}

1 ô, 1 오, Korea.

Sericinus telamon leechi subsp. nov.

o. Differs from $t$. montela Gray in the dark bands and patches being much narrower and less heavily marked.

ㅇ. Differs from $t$. montela in the light bands on the wings being much more extended and their yellow colour being much paler.

Habitat. Chang-yang (A. E. Pratt).

9 ơ ô, 7 우, Chang-Yang (A. E. Pratt), ex Leech coll. 


\section{Serecinus telamon montela Gray.}

Sericinus montela Gray, P.Z.S. 1852, p. 71 (Shanghai).

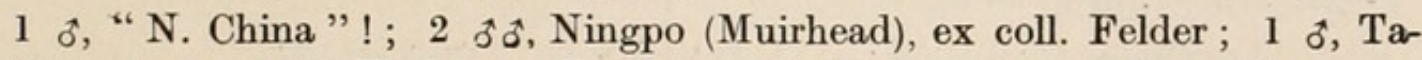
tsien-lu) ; 6 ธิ ठิ, 2 웅, Kiukiang, June 1887 (A. E. Pratt), ex coll. Leech.

\section{ZERYNTHIA.}

This genus is much better known as Thais Fabr., but Fabricius' name is preoccupied by Thais Bolt, 1798, a genus of Molluscs, so the next oldest name Zerynthia Ochs. (Ochsenheimer and Treitschke Schmetterlinge Europäis., 1816) must be used.

\section{Zerynthia cerisyi God.}

There are 8 subspecies of this species.

\section{Zerynthia cerisyi cerisyi (God.).}

Thais cerisyi Godart, Mém. Soc. Linn. Paris, vol. ii. p. 234. pl. 20. ff. 3, 4 (1822).

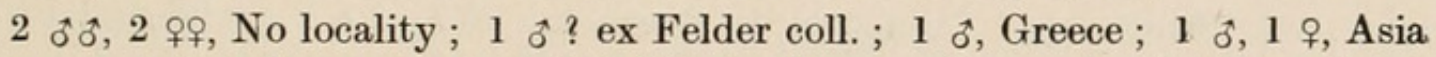

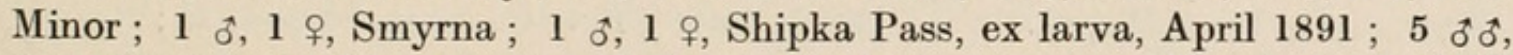
1 ㅇ, Burnabad, Smyrna; 6 ôิ 4 우, Cordelio, Smyrna, March-April 1905 (Dr. Martin) ; 2 ธิ oే, 2 우, Balkan Peninsula (Haberhauer), ex coll. Felder ; 1 ơ, Brussa (Mann), ex coll. Feld.

\section{Zerynthia cerisyi cypria Stich.}

Zeryntiia cerisyi cypria Stichel, Entom. Zeitschr. Stuttg. vol. xxi. p. 178 (1907) (Cyprus).

34 oิ $\hat{\sigma}^{2}, 20$ 우, Aghirda, foot of Kyrenia Range, Cyprus, 1,400 ft., March 1914 (G. F. Wilson) ; 1 क, St. Hilarion, Northern Range, Cyprus, 1,800 ft., April 1914 (G. F. Wilson), ex coll. A. E. Gibbs.

\section{Zerynthia cerisyi martini (Fruhst.).}

Thais cerisyi martini Fruhstorfer, Soc. Entom. vol. xxi. p. 147 (1906) (Rhodes).

5 ơ, Rhodes Island, March-April 1905 (Dr. Martin).

\section{Zerynthia cerisyi speciosa Stich.}

Zerynthia cerisyi speciosa Stichel, Entom. Zeitschr. Stuttg. vol. xxi. p. 83 (1907) (Syria, Palestine).

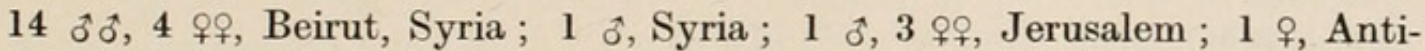
ochia ; 2 ơ , Lebanon, ex coll. Felder; 17 larvae, Beirut, Syria.

\section{Zerynthia cerisyi caucasica (Led.).}

Thais cerisyi var. caucasica Lederer, Wien. Entom. Mon. vol. viii. p. 165 (1864) (Caucasus).

2 ô่, 2 ำ, Caucasus ; 1 ô, 3 우, Cau asus (Haberhauer), ex coll. Felder.

Zerynthia cerisyi deyrollei (Oberth.).

Thais deyrollei Oberthur, Petites Nouv. Entom. vol. i. No. 2. p. 7 (1869) (Alpes-Pontiques, Asia Minor).

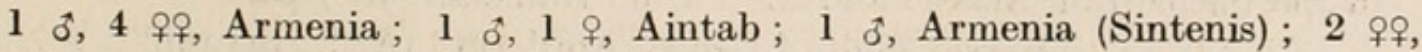

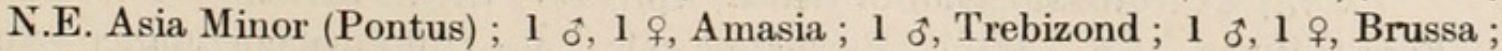

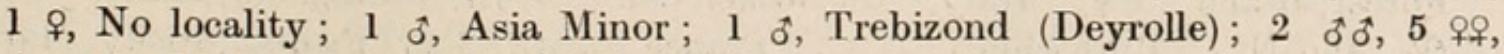
Amasia (Mann), ex coll. Felder. 


\section{Zerynthia cerisyi cretica (Rebel).}

Thais cerisyi var. cretica Rebel, Verh. Zool. Bot. Gesell. Wien. vol. liv. p. 2 (1904) (Crete).

2 ơ ô, Crete, May 1903; 1 ơ, Hag Myron, Crete (Holtz).

Zerynthia cerisyi louristana (Le Cerf).

Thxis cerisyi var. louristana Le Cerf, Bull. Soc.-Entsm.-France, 1908, p. 21 (Louristan).

1 ô, 1 \%, Sultanabad, S. Persia, 2,000 metres =6,500 ft., April 1910 (C. H. Ragnow); 1 o, 1 \%, Azuh, Louristan, Persia, April 1908.

Zerynthia polyxena (Schiff. and Den.).

Zerynthia polyxena polyxena (Schiff. and Den.).

Papilio polyxena Schiffermiller and Denis, Syst. Verz. Schmett. Wien, p. 162. No. 1. (1771) (Vienna).

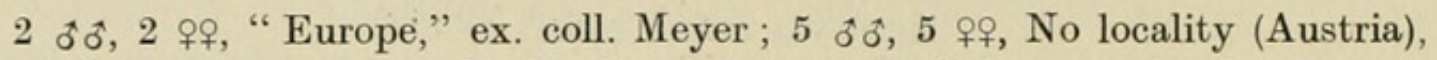
ex coll. Felder ; 4 ธิ oิ, 6 우, No locality ; 2 oิ oิ, 2 우, " Germany" ! bred 1894 ; 1 ô, 1 ㅇ, Brünn; 1 ô, Hungary, ex Buchecker coll.; 1 ô, 2 우, Hungary; 2 우, Kronstadt, Transylvania ; 1 ๙ै, Bishofsbad, Bihar Comitat, May 1911; 2 ๙ิ ô, 1 Juc, 1 Tiszovicza; nr. Szvinicza Costellitz, Hungary, May 1912; 18 ơ ô, 12 우, Csehtelek, Bihar Comitat, May 1912 ; 5 larvae ; 2 pupae ; eggs ; 2 Hymenopterous, and 1 Dipterous parasite.

\section{ab. rufescens Oberth.}

3 ธึఠ, Gravosa, S. Dalmatia, April 1901.

\section{Zerynthia polyxema subsp. ?}

I have 1 from Delphi (Krüper), which is very small and pale, and differs a good deal from $p$. polyxena in pattern, but I cannot describe it from 1 ô only.

I also have 1 $\delta, 1$, labelled Parnassus which agree with above in marking. but are of the same colour as polyxena ab. rufescens.

\section{Zerynthia polyxena subsp. ?}

I have a $0 \hat{q}$ from Talysch, Caspian Sea, which are very large and very strongly marked. These also are coloured like ab. rufescens. The material is too insufficient to describe this form.

\section{Zerynthia polyxena creusa (Meig.).}

Thais creusa Meigen (demnosia Dahl MS.), System. Beschr. Eur. Schmstt. vol. i. p. 161 (1829).

Stichel in Wytsman's Genera Insectorum, Fasc. 59, p. 10, No. 2b, quotes Meigen's creusa as ex Dahl MS. and does so also for the second quotation, Mann, Stett. Entom. Zeit. vol. v. p. 359 (1854). This is wrong as Meigen's creusa = demnosia Dahl MS. and only Mann's creusa = creusa Dahl MS. It is very unfortunate that this mistake has arisen, for Meigen's name must stand. The 6 specimens from Alassio and the 1 from Rome are very pale.

2 ơ ô, 4 के Alassio, Gulf of Genoa, April 1910 (A. E. Britton) ; 1 \%, Marino Forest, Rome ; 1 o," "Italy," Buchecker coll. ; 4 ôे, Bordighera, March-April

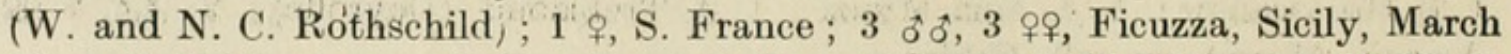
(Geo. C. Krüger), ex coll. Turati ; 1 ô, Zeng. Croatia, April 1912, ex coll. N. C. 
Rothschild; 1 o, 1 ㅇ, Florence (Steffanelli) ; 1 o, 2 우, Valescure, nr. St. Raphael, Var, April 1910 (W. Rothschild); 1 §. Antibes, Riviera, April 1904 .(W. Roth-

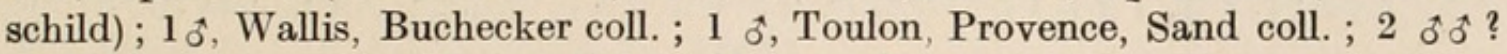

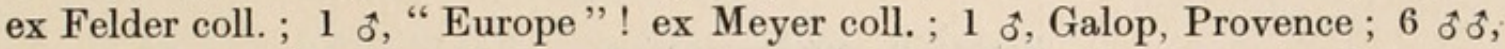
10 우, Hyères (H. Powell, March 1903, and Colonel N. Manders, April 1904); 1 s, 1 \&, Agaz, Dept. Var, April 1910 (W. Rothschild) ; 2 larvae, 2 pupa shells, 1 Hymenopterous parasite, Hyères (H. Powell).

\section{ab. vitrina ab. nov.}

This variety is very curious, the yellow is replaced by semitransparent dirty white and the red spots by yellow.

1 ô, Toulon, Sand coll. ; 1 ô, Agay, Dept. Var, April 1910 (W. Rothschild).

\section{Zerynthia polyxena subsp. ?}

Resembles creusa but much larger. The material is quite inadequate to describe this form.

1 ô, S. Russia; 1 ô, Ekaterinoslov, May 1882 (Bramson).

\section{Zerynthia polyxena latiaris Stich.}

Zerynthia polyxena latiaris Stichel in Wytsman, Genera Ins. Fase. 59. p. 11. (1907) (Central Italy, Alban Mountains).

$4 \hat{\jmath} \hat{\jmath}$, Mount Autore, Central Italy, 1,000 metres $=3250 \mathrm{ft}$., April 1909 (Geo. C. Krüger), ex coll. Turoti.

\section{Zerynthia rumina (Linn.).}

Herr Stichel in Wytsman's Genera Insectorum, Fasc. 59, p. 11, describes, under the name rumina andalusiana, a form of rumina in which the red on the forewing is more or less obsolescent and the black markings very heavy, while the red on the hindwing is reduced. This he records from Andalusia, especially Gibraltar (Type Gibraltar, No. 3113, Kgl. Zoological Museum, Berlin). I possess a number of such specimens from South Portugal, but with them were taken in great numbers quite typical rumina. From Gibraltar I have 6 specimens taken by Captain Jacobs, and I saw in his collection many more, all of which have very extended red markings. It is therefore quite evident that andalusiana Stichel can only be considered as an aberration.

\section{Zerynthia rumina rumina (Linn.).}

Papilio rumina Linnaeus, Syst. Nat. (Edit. x.) p. 480. No. 132. (1758) (South Europe).

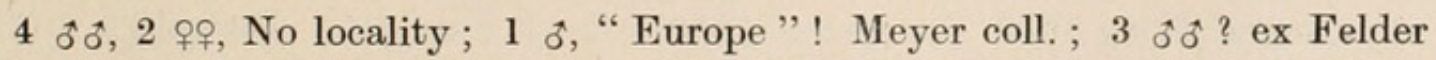
coll. ; 1 ô, 1 우, South Spain ; 5 oิ ô, 1 우, Gibraltar, February-March, 19101911 (Captain J. J. Jacobs) ; 12 oో $\sigma^{*} 4$ 우, Algeciras, March 1908 (N. Charles

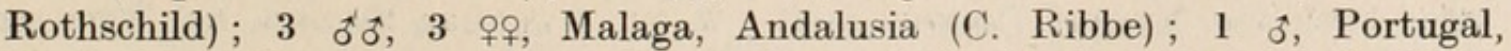
Buchecker coll. ; 4 ô ô, 2 우, Cintra, Portugal, April 1909-1910 (N. C. Rothschild and Dr. K. Jordan); 10 ô $\delta^{-} 6$ 우, Monchique, Algarve, Portugal, May 1910, 9 ठิ ๙ै, 9 우, Caldas de Monchique, April 1910 (Dr. K. Jordan) ; 3 larvae, Andalusia ; 1 larva, Monchique, May 12, 1910 (Dr. K. Jordan). 
ab. canteneri Stdgr.

2 우 ? ; 1 oิ, 1 우 ? ex Felder coll. ; 1 ઈ, 2 우, Malaga, Andalusia (C. Ribbe).

\section{Zerynthia rumina castiliana (Rühl).}

Thais rumina var. castiliana Rühl (and Heyne), Palaearct. Grossschmett. vol. i. p. 91 (1892) (Castile).

The one specimen from San Ildefonso is a strongly marked example of the aberration andalusiana Stich., showing that this aberration occurs also in other races of rumina.

2 ơ ơ, San Ildefonso, Segovia, Spain, June-August 1906 (M. de la Escalera); 2 우, Castile.

\section{Zerynthia rumina subsp. ?}

These two specimens are coloured like ab. canteneri, but the material is too scanty for description.

$1 \hat{o}, 1$ \&, Talysch, Caspian Sea.

\section{Zerynthia rumina medesicaste (Illig.).}

Papilio medesicaste Illiger, Maj. Insek:, vol. ii. p. 181 (1803) (South of France).

1 oै, Valescure, nr. St. Raphael, April 1910 (W. Rothschild) ; 6 ơ ô, 4 우, Hyères, April 1904 (Colonel N. Manders) ; 4 ô ô, 5 우우 ?; 2 ô ô, "Europe "! ex Meyer coll.; 2 $\widehat{\jmath}$, S. France, ex Buchecker coll.; 1 \&, S. France, Sand

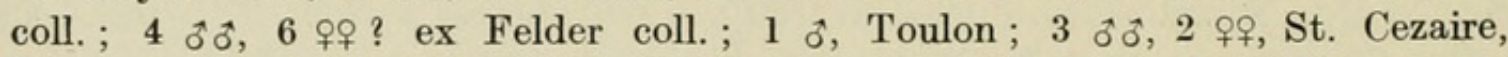
Basses Alpes, April 1910; 4 ơ $\widehat{o}$, St. Vincent, Digne Basses Alpes, May, 1908 ; 1 ô, 1 ㅇ, Digne, May 1904 (Colonel N. Manders).

\section{ab. honnoratii Boisd.}

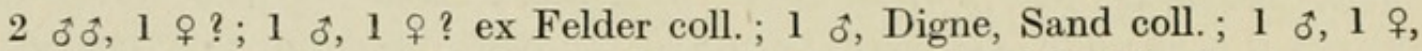
bred by Dr. Standfuss; 1 ô, Digne, May 4, 1904 (Colonel N. Manders).

\section{Zerynthia rumina mauretanica (Schulz).}

Thais rumina mauretanica Schulz, Intern. Entom. Zeitschr. Stuttg. vol. xxi. p. 267 (1908) (Morocco, Algeria).

1 oิ, 1 ㅇ, Tangiers; 1 ô, 1 ㅇ ? ; 9 ô ô, 3 우, Tlemcen, Prov. Oran, April 1913 (W. Rothschild and E. Hartert); 18 ôे, 4 우, Oran, April 1913 (W. Rothschild and E. Hartert) ; 1 \%, Santa Cruz, Oran, February, 1906 (Dr. Nissen); 3 ơ ô, 5 우, Hammam R'ihra; 2 ơ ơं, 2 우, April 1912 (W. Rothschild and K. Jordan); 1 ô, 1 \&, ex larva (larvae, May 1908 (W. Rothschild and K. Jordan), 1 emerged Tring 1909, the second 1910); 2 우, July 1916 (Faroult); 1 ot, 1 ㅇ, Blida les Glacières, June 1908 (W. Rothschild and K. Jordan) ; 26 ô ô, 19 çㅇ, Environs d'Alger (Hussein Dey, Maison Carrée, Femme Sauvage), March-April, 1905-1913 (Dr. Nissen, Captain Holl) ; 2 ơ ô, 2 우, Lac Fetzara, nr. Bône, ex larva emerged Tring, April 1910 (W. Rothschild and E. Hartert) ; 1 o, 1 \&, Ain Draham, Tunisia (Faroult); 3 larvae, Hammam R'ihra, May 1911 (W. Rothschild and E. Hartert); 1 larva, Lac Fetzara, May 1909 (W. Rothschild and E. Hartert). 
ab. distorta ab. nov.

This aberra ion has the hindwings cut square as if with scissors and generally has a row of red submarginal marks.

2 우, Ain Draham, Tunisia, March 1911 (Faroult).

ab. derubescens Schultz.

1 f, Alger, 1898 (E. Deschanges).

ab. canteneri Stdgr.

1 \&, Oran, April 20, 1913 (W. Rothschild and E. Hartert) ; 1 \&, Blida les Glacières, June 9, 1908 (W. Rothschild and K. Jordan). 


\section{$2 \mathrm{BHL}$ Biodiversity Heritage Library}

Rothschild, Lionel Walter Rothschild. 1918. "Catalogue of Zerynthiinae and allied genera in the Tring Museum, with critical notes." Novitates zoologicae : a journal of zoology in connection with the Tring Museum 25, 64-75. https://doi.org/10.5962/bhl.part.29761.

View This Item Online: https://www.biodiversitylibrary.org/item/24178

DOI: https://doi.org/10.5962/bhl.part.29761

Permalink: https://www.biodiversitylibrary.org/partpdf/29761

\section{Holding Institution}

Natural History Museum Library, London

\section{Sponsored by}

Natural History Museum Library, London

\section{Copyright \& Reuse}

Copyright Status: Public domain. The BHL considers that this work is no longer under copyright protection.

This document was created from content at the Biodiversity Heritage Library, the world's largest open access digital library for biodiversity literature and archives. Visit BHL at https://www.biodiversitylibrary.org. 